\title{
Various Types of Plaque Disruption in Culprit Coronary Artery Visualized by Optical Coherence Tomography in a Patient With Unstable Angina
}

\author{
Takashi Tanimoto, MD; Toshio Imanishi, MD; Atsushi Tanaka, MD; Takashi Yamano, MD; \\ Hironori Kitabata, MD; Shigeho Takarada, MD; Takashi Kubo, MD; Nobuo Nakamura, MD; \\ Kumiko Hirata, MD; Masato Mizukoshi, MD; Takashi Akasaka, MD
}

\begin{abstract}
A 58-year-old man underwent cardiac catheterization for unstable angina. The coronary angiogram revealed severe stenosis of the right coronary artery. Although $20-\mathrm{MHz}$, phased-array intravascular ultrasound (IVUS) only visualized eccentric, low echoic plaque at the culprit site, optical coherence tomography (OCT) clearly revealed ruptured plaque and an intraluminal thrombus. OCT also revealed a small ruptured plaque and an eroded plaque with intraluminal thrombi in a distal site remote from the culprit lesion, neither of which was visualized by IVUS. (Circ J 2009; 73: 187-189)
\end{abstract}

Key Words: Optical coherence tomography; Plaque disruption; Unstable angina

$\mathbf{P}$ aque rupture and subsequent thrombus formation is currently recognized as the most important mechanism of acute coronary syndrome (ACS)! Several intravascular ultrasound (IVUS) studies have evaluated the incidence and clinical presentation of plaque rupture in patients with $\mathrm{ACS}_{-}^{2-4}$ but pathological investigations have revealed that a non-rupture etiology, namely plaque erosion, also accounts for an important substrate of coronary thrombosis in patients who die following acute myocardial infarction: 5

Optical coherence tomography (OCT) is a new intravascular imaging modality with a high resolution of approximately $10-20 \mu \mathrm{m}$, which is 10 -fold higher than that of IVUS. We present a very unique case of unstable angina showing various types of plaque disruption in the culprit coronary artery.

\section{Case Report}

A 58-year old man was admitted to hospital because of new onset chest pain at rest. He had some coronary risk factors including diabetes mellitus, hypertension, and hypercholesterolemia. Aspirin, pravastatin sodium, and voglibose were administered before his admission. His total cholesterol and low-density lipoprotein cholesterol levels were $257 \mathrm{mg} / \mathrm{dl}$ and $179 \mathrm{mg} / \mathrm{dl}$, respectively. Fasting plasma glucose level was $101 \mathrm{mg} / \mathrm{dl}$ and hemoglobin Alc was $6.1 \%$. The level of high-sensitivity C-reactive protein was $0.9 \mathrm{mg} / \mathrm{L}$.

The coronary angiogram revealed $90 \%$ stenosis in the

(Received September 2, 2007; revised manuscript received March 18, 2008; accepted April 13, 2008; released online November 11, 2008) Department of Cardiovascular Medicine, Wakayama Medical University, Wakayama, Japan

No grant.

Mailing address: Takashi Tanimoto, MD, Department of Cardiovascular Medicine, Wakayama Medical University, 811-1 Kimiidera, Wakayama 641-8509, Japan. E-mail: taka-t@ wakayama-med.ac.jp All rights are reserved to the Japanese Circulation Society. For permissions, please e-mail: cj@j-circ.or.jp distal portion of the right coronary artery (RCA) (Fig 1). Before any coronary intervention, we performed 2.9-F, 20$\mathrm{MHz}$, phased-array IVUS (Eagle Eye Gold ${ }^{\circledR}$, Volcano Therapeutics, Rancho Cordova, CA, USA) and OCT (Image Wire ${ }^{\circledR}$, Light-lab imaging, Goodman, Co, Ltd, Nagoya, Japan) examinations with an automatic pull-back device from the distal portion at $1 \mathrm{~mm} / \mathrm{s}$.

IVUS showed only eccentric and low-echoic plaque with no evidence of rupture at the culprit site. OCT, however, revealed a tear of the fibrous cap with ulceration into the lipid-rich plaque (Fig 2A).

Furthermore, in a distal site of the RCA, a small ruptured plaque with thin fibrous cap was demonstrated by OCT (Fig 2B). The thickness of the fibrous cap was approximately $50 \mu \mathrm{m}$ and there was not an intraluminal thrombus, but remote from this lesion, an eroded plaque and intraluminal thrombi protruding into the lumen with an acoustic shadow were documented by OCT (Fig 2C). IVUS failed to

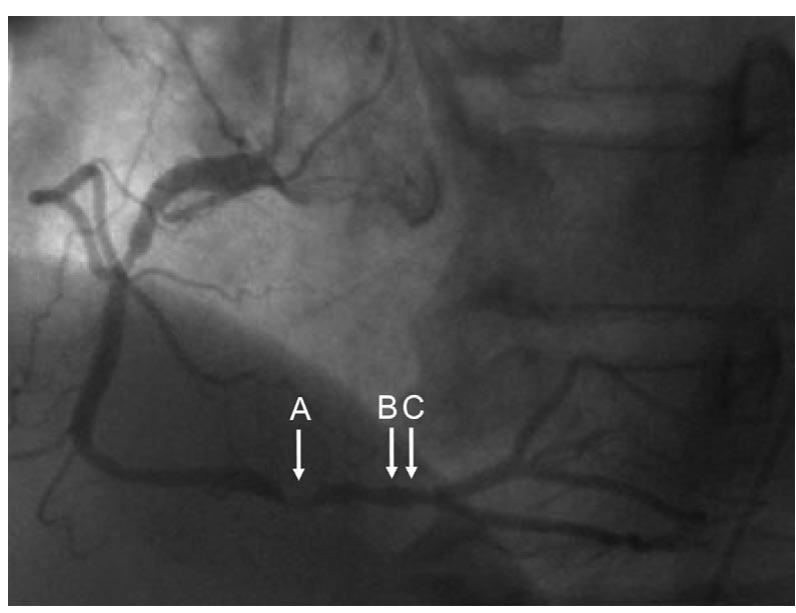

Fig 1. Coronary angiogram of the right coronary artery shows a severe stenosis in segment 3 (site A). 

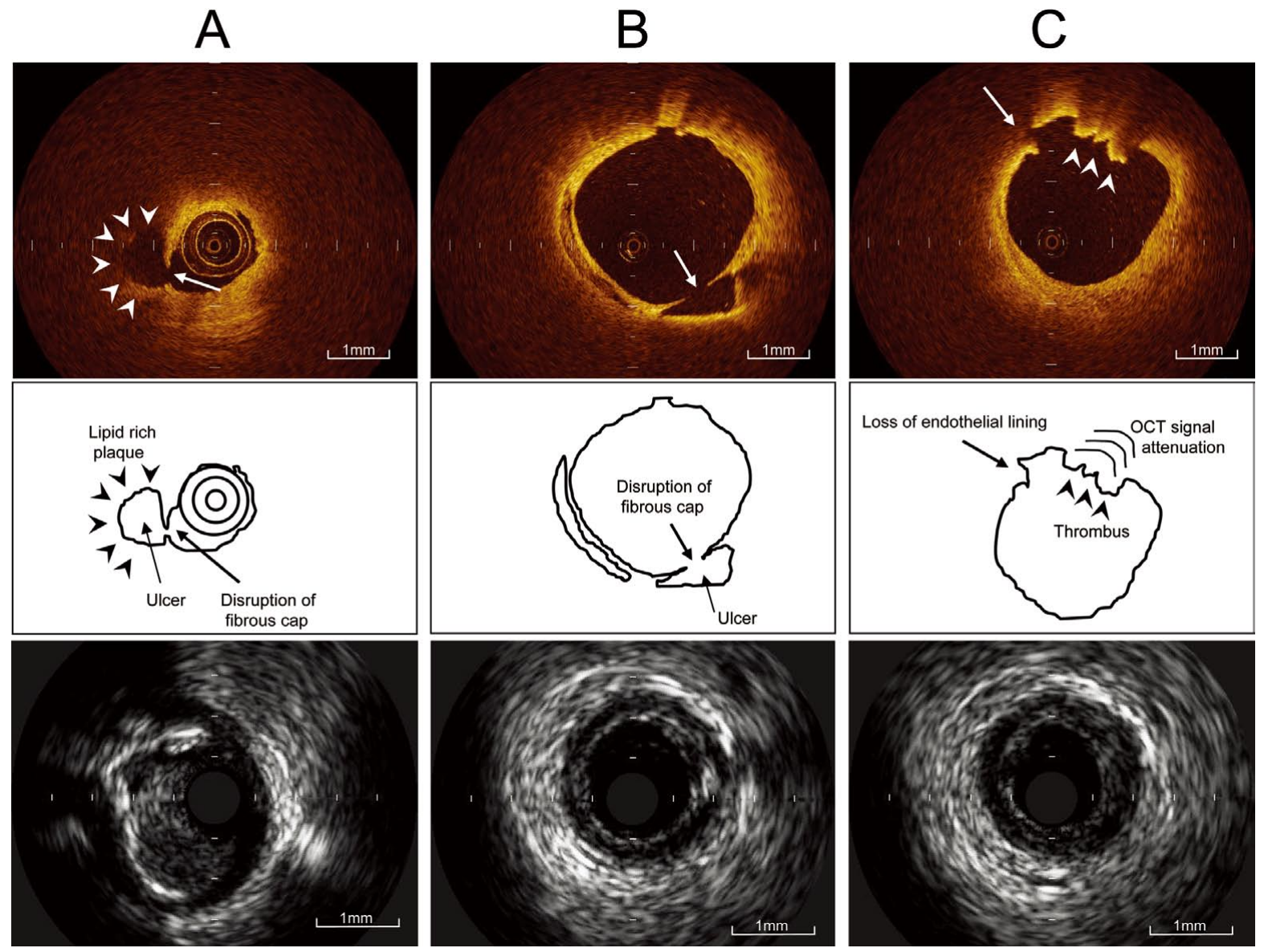

Fig 2. Optical coherence tomography (OCT: Upper), schematic of OCT (Middle), and intravascular ultrasound (IVUS) images (Lower) of site A (culprit lesion, B, and C). (A) Plaque rupture (arrow) with ulcer formation into the lipid-rich plaque (arrowhead) as seen with OCT. Eccentric, low-echoic plaque was documented by IVUS at the same site. (B) Small plaque rupture (arrow) with thin fibrous cap $(\approx 50 \mu \mathrm{m}$ thickness) remote from the culprit lesion. There is no intraluminal thrombus. Silent plaque rupture was suspected. (C) Plaque erosion (arrow) with intraluminal thrombi with acoustic shadow (arrowhead) as observed with OCT.

detect these lesions.

We also evaluated the proximal segment of the RCA, including the stenosis in segment 2 , by IVUS and OCT. IVUS showed eccentric plaque and OCT demonstrated only fibrous plaque without evidence of thin cap fibroatheroma (TCFA) or plaque disruption, and there were no vulnerable lesions in the proximal RCA.

\section{Discussion}

In this case, OCT clearly revealed an event-related ruptured plaque, a small silent ruptured plaque, and an eroded plaque with thrombi in the culprit coronary artery. To the our best of knowledge, this is the first report of OCT detecting plaque ruptures with/without thrombus and an eroded plaque with thrombus in the same coronary artery in vivo.

OCT is thought to be a promising imaging device for the evaluation of the vulnerability of plaque that is predominantly superficial in location? Raffel and Jang reported a case of stable angina caused by ruptured TCFA plaque evaluated by OCT? In the present case, various types of plaque disruption were identified by OCT and we could observe the morphology of both culprit lesion and non-culprit lesions (event-unrelated plaque disruption and erosion) in the same coronary artery before any coronary intervention.
Multiple plaque ruptures have been reported in prior angiographic studies of patients with ACS 9,10 Several IVUS studies also reported that ruptured plaques were identified in non-culprit lesions in $20-79 \%$ of patient with ACS?-4 Those findings support the concept of multifocal plaque instability in patients with ACS. Because OCT can clearly detect small ruptured plaque, multiple plaque rupture may be documented more frequently by OCT than with IVUS. In order to prevent further events related to non-culprit lesions, systemic medical therapy is most important in the OCT era, even if we perform coronary intervention for the culprit lesion.

The clinical significance of plaque erosion has been unknown because IVUS cannot detect it in vivo. OCT may provide useful information about plaque erosion.

Intracoronary thrombus might have a critical role in the pathogenesis of ACS. Neither angiography nor IVUS can reliably demonstrate thrombus. We identified the mass shown in Fig 2C as red thrombus by OCT because it had signal attenuation behind the mass, as reported previously! 1

Current limitations of OCT are poor tissue penetration and interference by blood flow? IVUS is superior for evaluating vessel wall remodeling than OCT. Using both OCT and IVUS may help us to assess plaque morphology, including the vessel wall, and thus enable detection of 
various types of plaque disruption with clear images in patients with ACS.

\section{References}

1. Fuster V, Moreno PR, Fayed ZA, Corti R, Badimon JJ. Atherothrombosis and high-risk plaque. J Am Coll Cardiol 2005; 46: 937-954.

2. Rioufol G, Finet G, Ginon I, André-Fouët X, Rossi R, Vialle E, et al. Multiple atherosclerotic plaque rupture in acute coronary syndrome: A three-vessel intravascular ultrasound study. Circulation 2002; 106: 804-808.

3. Hong MK, Mintz GS, Lee CW, Kim YH, Lee SW, Song JM, et al. Comparison of coronary plaque rupture between stable angina and acute myocardial infarction: A three-vessel intravascular ultrasound study in 235 patients. Circulation 2004; 110: $928-933$.

4. Tanaka A, Shimada K, Sano T, Namba M, Sakamoto T, Nishida Y, et al. Multiple plaque rupture and C-reactive protein in acute myocardial infarction. J Am Coll Cardiol 2005; 45: 1594-1599.

5. van der Wal AC, Becker AE, van der Loos CM, Das PK. Site of intimal rupture or erosion of thrombosed coronary atherosclerotic plaques is characterized by an inflammatory process irrespective of the dominant plaque morphology. Circulation 1994; 106: 36-44.

6. Arbustini E, Dal Bello B, Morbini P, Burke AP, Bocciarelli M, Specchia $\mathrm{G}$, et al. Plaque erosion is a major substrate for coronary thrombosis in acute myocardial infarction. Heart 1999; 82: 269-272.

7. MacNeill BD, Lowe HC, Takano M, Fuster V, Jang IK. Intravascular modalities for detection of vulnerable plaque; current status. Arterioscler Thromb Vasc Biol 2003; 23: 1333-1342.

8. Raffel OC, Jang IK. Incidental finding of ruptured thin-cap fibroatheroma by optical coherence tomography. Eur Heart J 2006; 27: 2393.

9. Chester MR, Chen L, Kaski JC. The natural history of unheralded complex coronary plaques. J Am Coll Cardiol 1996; 28: 604-608.

10. Goldstein JA, Demetriou D, Grines CL, Pica M, Shoukfeh M, O'Neill WM. Multiple complex coronary plaques in patients with acute myocardial infarction. N Engl J Med 2000; 343: 915-922.

11. Kume T, Akasaka T, Kawamoto T, Ogasawara Y, Watanabe N, Toyota E, et al. Assessment of coronary arterial thrombus by optical coherence tomography. Am J Cardiol 2006; 97: 1713-1717. 